\title{
SITUACIÓN NUTRICIONAL DE LOS NIÑOS MIGRANTES VENEZOLANOS A SU INGRESO AL PERÚ Y LAS ACCIONES EMPRENDIDAS PARA PROTEGER SU SALUD Y NUTRICIÓN
}

\author{
Rocío Vargas-Machuca ${ }^{1, a}$, Carlos E. Rojas-Dávila1,a, M. Michelle Jiménezi,b, \\ Carol R. Piscoya-Magallanes ${ }^{1, a}$, Hugo Razuri ${ }^{1, c}$, María Elena Ugaz ${ }^{1, a}$
}

\begin{abstract}
RESUMEN
La elevada migración venezolana ha mostrado ser un gran desafío para el Perú debido a las necesidades de atención que requieren los inmigrantes. De modo preocupante, los menores de cinco años son quienes se encuentran más vulnerables. A fin de conocer el estado nutricional de este grupo en tránsito migratorio al Perú, se realizó un estudio descriptivo con dos rondas de recolección de datos (agosto de 2018 y marzo de 2019) en el Centro Binacional de Atención Fronteriza de Tumbes. En cada ronda, a 322 y 619 niños venezolanos menores de cinco años se determinó su estado nutricional, morbilidad y características de la alimentación, así como las acciones de salud y nutrición implementadas al momento de su ingreso al Perú. Para cada ronda, la desnutrición aguda afectó al 3,2\% y 3,0\%, la desnutrición crónica al $16,5 \%$ y $17,9 \%$, la anemia al $34,8 \%$ y $25,0 \%$, la tos o dificultad para respirar estuvo presente en el $31,3 \%$ y $17,6 \%$, las diarreas en el $16,6 \%$ y $9,9 \%$. Cerca de siete de cada diez niños menores de dos años lactó el día previo. Durante el viaje migratorio y para cada ronda, el $58,3 \%$ y $47,0 \%$ de los niños menores de dos años aumentó la frecuencia de lactadas, la frecuencia mínima de alimentación (FMA) fueron alcanzadas sólo por el 13,9\% y 18,0\%. La reacción oportuna de atención en salud y nutrición frente al ingreso de la población migrante es un buen primer paso, el sistema de salud necesita generar las condiciones para que los servicios de salud puedan brindar la cobertura con la calidad adecuada a toda la población que reside en el territorio nacional, bajo el enfoque de que el derecho a la salud corresponde a todas las personas sin ningún tipo de exclusión.
\end{abstract}

Palabras clave: Migración Humana; Lactante; Preescolar; Estado Nutricional; Venezuela (fuente: DeCS BIREME).

\section{NUTRITIONAL SITUATION OF VENEZUELAN MIGRANT CHILDREN UPON ENTRY INTO PERU AND THE ACTIONS TAKEN TO PROTECT THEIR HEALTH AND NUTRITION}

\begin{abstract}
The high level of Venezuelan migration is a great challenge for Peru due to the healthcare needs of immigrants. Worryingly, children under the age of five are the most vulnerable. In order to know the nutritional status of this group in migratory transit to Peru, a descriptive study was carried out with two rounds of data collection (August 2018 and March 2019) at the Binational Border Healthcare Center in Tumbes. In each round, nutritional status, morbidity and food characteristics were determined for 322 and 619 Venezuelan children under the age of five, as well as any health and nutrition actions implemented upon their entry into Peru. For each round, acute malnutrition affected $3.2 \%$ and $3.0 \%$; chronic malnutrition $16.5 \%$ and $17.9 \%$; anemia $34.8 \%$ and $25.0 \%$; cough or shortness of breath was present in $31.3 \%$ and $17.6 \%$; diarrhea in $16.6 \%$ and $9.9 \%$. Nearly seven out of ten children under the age of two breastfed the day before. During the migratory journey and for each round, $58.3 \%$ and $47.0 \%$ of children under two years of age increased their frequency of breastfeeding, the minimum meal frequency (MMF) was met only by $13.9 \%$ and $18.0 \%$. While the timely reaction in terms of healthcare and nutrition facing the inflow of migrant population is a good first step, the health system needs to generate the conditions so that health services can provide coverage with adequate quality to the entire population residing in the national territory, under the premise that the right to health is for everybody without any type of exclusion.
\end{abstract}

Keywords: Human Migration; Infant; Child, Preschool; Nutritional Status; Venezuela (source: MeSH NLM).

1 Fondo de las Naciones Unidas para la Infancia (UNICEF). Lima, Perú.

a Licenciado(a) en nutrición; ${ }^{\mathrm{b}}$ Bachiller en nutrición, magíster en Ciencias de la Nutrición Humana; ${ }^{\mathrm{c}}$ médico cirujano, magíster en Salud Pública Recibido: 17/07/2019 Aprobado: 04/09/2019 En línea: 23/09/2019

Citar como: Vargas-Machuca R, Rojas-Dávila CE, Jiménez MM, Piscoya-Magallanes CR, Razuri H, Ugaz ME. Situación nutricional de los niños migrantes venezolanos a su ingreso al Perú y las acciones emprendidas para proteger su salud y nutrición. Rev Peru Med Exp Salud Publica. 2019;36(3):504-10. doi: http://dx.doi.org/10.17843/rpmesp.2019.363.4676. 


\section{INTRODUCCIÓN}

En décadas pasadas, Venezuela se caracterizó en ser un país receptor de migrantes, al 2010 contaban con más de un millón de migrantes ${ }^{(1)}$, quienes se movilizaron principalmente por razones laborales provenientes de países vecinos ${ }^{(2)}$. La grave crisis que afecta a Venezuela ha originado que cientos de miles de personas abandonen su país en busca de mejores oportunidades de vida, principalmente a países latinoamericanos. Además de las tradicionales razones de migración (económicas, conflictos, guerras, insatisfacción de los servicios e instituciones), se suman el bienestar de los niños como un fuerte conductor de las decisiones de migración ${ }^{(3)}$, por lo cual entre 20072016, los intentos y planes de migración fueron más altos en los hogares con presencia de menores de edad.

Los niños migrantes enfrentan diversos retos durante la travesía migratoria debido a que sus familias cuentan con limitaciones económicas que los fuerza a desplazarse por caminos pocos seguros y en condiciones precarias, muchos de ellos enfrentan la pérdida de la unidad familiar quedando a cargo de terceras personas. Los niños y sus familias enfrentan desafíos para regularizar su estatus migratorio, lo que afecta su acceso a la protección social, la salud, el desarrollo de la primera infancia, la educación, los medios de vida sostenibles y la protección de los niños ${ }^{(3)}$. La falta de políticas públicas integrales sobre temas de migración en los países de acogida hace que los niños corran un mayor riesgo de discriminación, violencia, xenofobia, explotación y abuso en los países de tránsito o de destino.

El objetivo de este artículo es describir el estado nutricional, morbilidad y características de la alimentación de los niños venezolanos migrantes menores de cinco años de edad, así como las acciones de salud y nutrición implementadas al momento de su ingreso al Perú.

\section{EL FLUJO MIGRATORIO VENEZOLANO EN LATINOAMERICA Y EL PERÚ}

Los países de América Latina y el Caribe albergan aproximadamente 3,2 millones de los cuatro millones de migrantes y refugiados venezolanos en todo el mundo, y los 800000 restantes se encuentran en otras regiones del mundo ${ }^{(4)}$. De acuerdo con informes del Fondo de las Naciones para la Infancia (UNICEF), se estima que más de un millón de niños en Brasil, Colombia, Ecuador, Guyana, Panamá, Perú y Trinidad y Tobago necesitarán asistencia en 2019 (5).

A enero del 2019, en un lapso de tres años ingresaron al Perú 680 mil ciudadanos venezolanos. De acuerdo con estimaciones realizadas en el control fronterizo de Tumbes en dos momentos, alrededor del $25 \%$ de los grupos de viaje que ingresan, lo hacen con menores de edad ${ }^{(6,7)}$. La mitad de esta población infantil inmigrante son menores de cinco años.

Como todo desplazamiento migratorio masivo, en especial los forzados por crisis sociales, políticas o naturales, es de esperar que ocurran consecuencias en el estado de salud, nutricional y emocional de las personas, especialmente en los niños.

\section{EL RETO DE CONTAR CON INFORMACIÓN SOBRE LA SALUD Y NUTRICIÓN INFANTIL PARA LA TOMA DE DECISIONES EN EL CONTEXTO DEL TRÁNSITO MIGRATORIO}

Contar con información oportuna y de calidad para la toma de decisiones en un contexto de rápido y creciente flujo migratorio es un gran desafío. En estos contextos la provisión de servicios desde el Estado suele verse sobrepasada generando déficit en la atención y comprometiendo su calidad.

Ante la necesidad de contar con la información del estado nutricional de los niños migrantes venezolanos menores de cinco años, para implementar o ajustar acciones específicas de salud y nutrición, la Dirección Regional de Salud (DIRESA) de Tumbes y UNICEF organizaron un estudio descriptivo en dos rondas de recolección de datos, en agosto de 2018 y marzo de 2019. Este estudio permitió evaluar el estado nutricional y las condiciones y entorno familiar de viaje, realizadas en el Centro Binacional de Atención Fronteriza (CEBAF) de Tumbes.

Por sus características, el flujo migratorio en la frontera hace muy difícil establecer un marco muestral definido. Sin embargo, dado que el CEBAF es un punto de espera obligatorio para realizar los trámites migratorios, es posible tener en el CEBAF a todas las personas en flujo migratorio disponibles para el estudio. Esto llevó a elegir el muestreo consecutivo, un método no probabilístico que en este caso incluyó a todos los niños menores de cinco años que llegaron al CEBAF en un rango de tiempo determinado ${ }^{(8,9)}$. La primera ronda fue del 22 al 29 de agosto de 2019 y en la segunda del 3 al 12 de marzo del 2019. Para ambas rondas, se consideró días consecutivos que comprendieran al menos un fin de semana, cada día tuvo un ciclo de recolección continua de 13 horas en la primera ronda (6:00 a 19:00 horas) y, 16 horas en la segunda (6:00 a 22:00 horas). De modo paralelo, se reclutó personal de salud capacitado con experiencia en estudios de base poblacional, quienes fueron estandarizados en mediciones antropométricas y dosaje de hemoglobina por el Centro Nacional de Alimentación y Nutrición (CENAN) y por UNICEF. La capacitación incluyó la administración 
del cuestionario y su registró en tabletas electrónicas o en papel, procedimientos y protocolos de ética, contención emocional y reconocimiento de situaciones de vulnerabilidad para la derivación a servicios ad-hoc. El lenguaje y comprensión del cuestionario fueron validados con ciudadanos venezolanos, los pilotos de cada ronda se realizaron en terminales terrestres en Tumbes con una profesional de salud y en promedio participaron diez personas en la validación.

El puesto de evaluación fue un ambiente aledaño al puesto de inmunizaciones de la DIRESA, paso previo y obligatorio para la gestión migratoria de toda persona que ingresa al Perú por el CEBAF, lo que permitió identificar y registrar a toda la población objetivo. A los padres y tutores de los niños se les explicó los objetivos y procedimientos del estudio y se obtuvieron sus consentimientos. Se realizaron entrevistas que duraron entre 20 a 30 minutos, luego se efectuaron las mediciones antropométricas y el dosaje de hemoglobina a los niños.

La entrevista fue realizada al cuidador del niño en quien se indagó sobre sus características socioeconómicas y en relación con los niños, sobre la situación de la lactancia y alimentación del día previo y la presencia de morbilidad (fiebre, diarrea y dificultades respiratorias) en los tres últimos días previos. En la segunda ronda se incorporó preguntas sobre cambios emocionales en los niños y síntomas de depresión en los cuidadores en los últimos 15 días, relacionados al proceso migratorio.

La medición del peso y talla se realizó según los protocolos recomendados por la OMS ${ }^{(10)}$. La concentración de hemoglobina fue medida a través de una muestra de sangre capilar y un analizador automatizado (HemoCue 201+ en la primera ronda y HemoCue $301+$ en la segunda). Todos los equipos fueron previamente calibrados. La administración del cuestionario, el registro de la información en tabletas electrónicas y las mediciones biológicas se hicieron en ambientes climatizados para evitar alteraciones de las lecturas dado el clima tropical que superaba los $30^{\circ} \mathrm{C}$.

El estado nutricional fue evaluado a través de los índices talla para la edad (T/E) y peso para la talla $(P / T)$, empleando los estándares de crecimiento infantil de la OMS ${ }^{(11)}$. La anemia fue definida como una concentración de hemoglobina inferior a $11 \mathrm{~g} / \mathrm{dL}$, de acuerdo con los criterios de OMS ${ }^{(12)}$. La frecuencia mínima de alimentación (FMA) ${ }^{(13)}$ se utilizó como un indicador de la calidad de alimentación del niño durante el viaje, según su edad y estado de lactancia (para los que lactan se considera el consumo en el día anterior, de un mínimo de dos preparaciones espesas o sólidas en las edades de seis a ocho meses y tres preparaciones entre nueve y 24 meses; en los no lactantes la frecuencia debe ser 4 veces al día). El rol de la lactancia durante la travesía migratoria se evaluó en función si había variado o cesado.
El control de calidad de la encuesta fue diario en ambas rondas, los encuestadores verificaron la información y enviaron sus formatos cada dos días a un supervisor. En la segunda ronda el envío fue vía internet a un servidor. En ambas rondas, la captura electrónica contó con programación de alertas para datos inconsistentes $u$ omisos. La información se procesó en el programa IBM SPSS Statistics for Windows, versión 23 (IBM Corp., Armonk, N.Y., USA), los índices antropométricos se procesaron en el programa Anthro V3.2 ${ }^{(14)}$.

Todas las evaluaciones contaron con consentimiento verbal de los padres o tutores de los niños, en la segunda ronda estos fueron grabados. Los resultados nutricionales fueron entregados por escrito a los padres o cuidadores, quienes recibieron orientaciones y derivados inmediatamente a una siguiente atención en donde recibieron atención y consejería especializada en salud y nutrición materna e infantil.

\section{LA SITUACIÓN NUTRICIONAL, MORBILIDAD, CONDICIONES DE VIAJE DE LOS NIÑNOS MIGRANTES VENEZOLANOS MENORES DE CINCO AÑOS}

Al conjunto de personas, familiares o no, que arribaron juntas al Perú se les denominó «grupo de viaje». En las rondas 1 y 2 se entrevistó a 287 y 537 grupos de viaje con 322 y 619 niños venezolanos menores de cinco años, respectivamente. Por cada ronda, las medias de edad en meses de los niños fueron $26,4 \pm 16,6$ y $26,4 \pm 15,6$, mientras, el $52,8 \%$ y $49,4 \%$ fueron varones.

\section{ESTADO NUTRICIONAL Y MORBILIDAD}

Los niños migrantes afectados de desnutrición aguda (DA) mostraron prevalencias similares en ambas rondas, 3,2 y 3,0\% respectivamente; la desnutrición crónica (DC) afectó al $16,5 \%$ y $17,9 \%$ y la anemia al $34,8 \%$ y $25,0 \%$. La enfermedad diarreica en la ronda 1 afectó al 16,6\% y en la ronda 2 al 9,9\%. La tos o dificultades para respirar fue la morbilidad más reportada por los cuidadores, 31,3\% y $17,6 \%$, respectivamente para cada ronda; mientras que, la fiebre afectó al 14,4\% y 10,1\%. Al considerar alguno de los tres signos, el $44,1 \%$ y $29,5 \%$ de los niños los presentó en los tres últimos días a su arribo al país (Tabla 1).

\section{CARACTERÍSTICAS DEL CUIDADOR}

En ambas rondas, los cuidadores de los niños predominantemente fueron sus madres (85,1\% y 92,9\%), en la ronda 1 la media de edad fue $28,1 \pm 7,4$ años, en la ronda 2 fue $27,1 \pm 17,1$ años. Los cuidadores con 18 años o menos fueron el $3,1 \%$ y $5,4 \%$, las gestantes fueron el 
Tabla 1. Estado nutricional y morbilidad de niños inmigrantes venezolanos menores de cinco años.

\begin{tabular}{|c|c|c|c|c|}
\hline \multirow{2}{*}{$\begin{array}{l}\text { Condiciones según } \\
\text { edades en meses }\end{array}$} & \multicolumn{2}{|c|}{ Ronda 1} & \multicolumn{2}{|c|}{ Ronda 2} \\
\hline & $\mathbf{n}^{\dagger}$ & $\%^{\ddagger}$ & $\mathbf{n}^{\dagger}$ & $\% \neq$ \\
\hline \multicolumn{5}{|l|}{ Desnutrición crónica* } \\
\hline $0-5$ & 21 & 19,0 & 45 & 6,7 \\
\hline $6-11$ & 56 & 14,3 & 82 & 18,3 \\
\hline $12-23$ & 79 & 19,0 & 174 & 23,5 \\
\hline $24-35$ & 57 & 19,3 & 136 & 22,7 \\
\hline $36-47$ & 60 & 18,3 & 100 & 12,0 \\
\hline $48-59$ & 43 & 7,0 & 77 & 10,5 \\
\hline Total & 316 & 16,5 & 614 & 17,9 \\
\hline \multicolumn{5}{|l|}{ Desnutrición aguda* } \\
\hline $0-5$ & 22 & 0,0 & 45 & 0,0 \\
\hline $6-11$ & 56 & 0,0 & 82 & 3,7 \\
\hline $12-23$ & 79 & 5,1 & 174 & 4,7 \\
\hline $24-35$ & 57 & 1,8 & 136 & 1,5 \\
\hline $36-47$ & 60 & 3,3 & 100 & 1,0 \\
\hline $48-59$ & 43 & 7,0 & 77 & 5,3 \\
\hline Total & 317 & 3,2 & 614 & 3,0 \\
\hline \multicolumn{5}{|l|}{ Anemia** } \\
\hline $0-5$ & 13 & 38,5 & 21 & 0,0 \\
\hline $6-11$ & 46 & 58,7 & 76 & 55,3 \\
\hline $12-23$ & 67 & 44,8 & 176 & 36,9 \\
\hline $24-35$ & 49 & 28,6 & 135 & 16,3 \\
\hline $36-47$ & 54 & 18,5 & 99 & 11,1 \\
\hline $48-59$ & 35 & 17,1 & 77 & 7,8 \\
\hline Total & 264 & 34,8 & 584 & 25,0 \\
\hline \multicolumn{5}{|l|}{ Morbilidad reportada" } \\
\hline Diarrea $^{*}$ & 320 & 16,6 & 614 & 9,9 \\
\hline $\begin{array}{l}\text { Tos o dificultad para } \\
\text { respirar }\end{array}$ & 320 & 31,3 & 614 & 17,6 \\
\hline Fiebre & 320 & 14,4 & 614 & 10,1 \\
\hline Alguna patología & 320 & 44,1 & 614 & 29,5 \\
\hline
\end{tabular}

* Definido como Z-score < -2 SD de la mediana para talla por edad (desnutrición crónica) y peso para la talla (desnutrición aguda), estándares de crecimiento de la Organización Mundial de la Salud 2006.

** Concentraciones de hemoglobina menor a $11 \mathrm{~g} / \mathrm{dL}$.

"Últimos tres días, reportado por la madre.

* Tres o más deposiciones líquidas o semilíquidas.

† Tamaño de la muestra para cada categoría.

‡ Proporción estimada de la condición.

$5,4 \%$ y $4,1 \%$ de las mujeres. Cuatro de cada diez mujeres se encontraban dando de lactar, $42 \%$ en la ronda $1 \mathrm{y}$ $41,5 \%$ en la ronda 2 . En cuanto al nivel educativo, $10,8 \%$ y $15,1 \%$ de los cuidadores del niño contaban con estudios primarios o no tenían ningún estudio, respectivamente para cada ronda. La proporción con niveles educativos más altos, técnico o universitario, pasó de $44,3 \%$ a $28,7 \%$. En ambas rondas, el $45 \%$ manifestó haber tenido un empleo o trabajo por cuenta propia antes de salir de su país de origen (Tabla 2).

\section{CONDICIONES DEL VIAJE}

En ambos momentos, la razón más frecuente de migración mencionada por los cuidadores de los niños fue la económica, $62,4 \%$ y 42,2\%. El acceso a alimentos en la ronda 1 fue manifestado por el 7,5\%, proporción que se triplicó a $22,7 \%$ en la ronda 2 . La reunificación familiar mantuvo proporciones cercanas, $21,9 \%$ y $20,8 \%$. El tipo de grupo de viaje predominante fue el familiar, cuando además del niño y su cuidador se hallan otros parientes, en la ronda 1 fue en el $75,5 \%$ y $60,1 \%$ en la ronda 2 . El porcentaje de la modalidad «Solo cuidador y niño» incrementó de 12,5\% a $21,7 \%$. El viaje desde la salida de Venezuela hasta Perú tuvo una mediana de duración de cinco días en ambas rondas, el porcentaje de los que les tomó un mes o más para arribar a Perú pasó de $9,0 \%$ y $17,6 \%$ entre ambas rondas (Tabla 2).

\section{CARACTERISTIICAS DE LA LACTANCIA Y ALIMENTACIÓN EN LOS NIÑOS MENORES DE 2 AÑOS.}

En la ronda 1 y en la ronda 2 las cuidadoras manifestaron que el $68,8 \%$ y $71,7 \%$ de sus hijos respectivamente, habían lactado el día anterior a la encuesta. Respecto de la frecuencia de la lactancia, en la ronda 1 el 58,3\% de las cuidadoras manifestaron que la aumentaron y el $27,5 \%$ que la mantuvieron; en la ronda 2 estas mismas prácticas fueron reportadas en el $47,0 \%$ y $34,0 \%$ respectivamente. Con relación a FMA en la ronda 1, el 13,8\% de los niños recibió alimentos sólidos o semisólidos en la frecuencia esperada, mientras que en la ronda 2 lo recibió el 18\% (Tabla 3).

\section{ACCIONES PARA PROTEGER LA NUTRICIÓN Y EL ESTADO DE SALUD DE LOS NIÑOS MIGRANTES}

Al no tener estadísticas oficiales actuales del estado nutricional de los niños en Venezuela ${ }^{(15)}$, no es posible aproximar en qué medida el tránsito migratorio ha impactado en la nutrición de estos niños. Sin embargo, se observa que la FMA fue alcanzada por menos de la quinta parte de los niños, es decir los niños en su mayoría no reciben alimentos sólidos o semisólidos la cantidad de veces recomendada que ocurriría muy probablemente por las difíciles condiciones económicas y logísticas del viaje de tránsito migratorio. Asimismo, las cuidadoras estarían recurriendo a incrementar la frecuencia de lactadas frente a este hecho.

Por otro lado, aunque en general el porcentaje de niños con DA estuvo alrededor del $3 \%$, en el subgrupo de niños en el rango de edad de 48 a 59 meses, este porcentaje alcanza o es mayor a $5 \%$, punto a partir del cual se considera que habría un problema de salud pública; la alimentación y las morbilidades reportadas estarían asociadas a esta condición. Sin bien es cierto que este subgrupo representa una proporción relativamente menor del total de niños de la muestra (13,6\% ronda 1 y $12,5 \%$ ronda 2$)$, es un hecho llamativo ya que las cuidadoras luego de su ingreso al país no tendrían de manera inmediata los medios para mejorar las condiciones de alimentación y cuidado en general de los niños. 
Tabla 2. Características de los cuidadores de los niños inmigrantes venezolanos menores de cinco años y del viaje de migración.

\begin{tabular}{|c|c|c|}
\hline \multirow[t]{2}{*}{ Características } & $\begin{array}{c}\text { Ronda } 1 \\
(\mathrm{n}=287)\end{array}$ & $\begin{array}{c}\text { Ronda } 2 \\
(n=537)\end{array}$ \\
\hline & $\%$ & $\%$ \\
\hline \multicolumn{3}{|l|}{ Del cuidador } \\
\hline Edad en años [promedio (DE)] & $28,1(7,4)$ & $27,1(17,1)$ \\
\hline Mujeres & 91,3 & 96,5 \\
\hline Adolescentes & 3,1 & 5,4 \\
\hline Embarazadas (\% mujeres) & 5,4 & 4,1 \\
\hline Mujeres lactantes ( $\%$ mujeres) & 42,0 & 41,5 \\
\hline Casado o conviviente & 71,1 & 96,3 \\
\hline \multicolumn{3}{|l|}{ Relación con el niño } \\
\hline Madre & 85,9 & 93,1 \\
\hline Padre & 6,3 & 3,2 \\
\hline Familiar/otro & 7,8 & 3,7 \\
\hline \multicolumn{3}{|l|}{ Nivel de educación } \\
\hline Ninguno & 1,7 & 0,6 \\
\hline Primario & 9,1 & 14,5 \\
\hline Secundario & 44,9 & 56,2 \\
\hline Técnico superior & 15,7 & 14,7 \\
\hline Universitario & 28,6 & 14,0 \\
\hline \multicolumn{3}{|l|}{$\begin{array}{l}\text { Condición de actividad antes de } \\
\text { migrar }\end{array}$} \\
\hline Empleado/a & 30,3 & 32,2 \\
\hline Sin actividad & 52,3 & 50,3 \\
\hline Estudiante & 2,4 & 4,1 \\
\hline Trabajador por cuenta propia & 14,6 & 13,2 \\
\hline Retirado o jubilado & 0,3 & 0,2 \\
\hline \multicolumn{3}{|l|}{ Razones de migración } \\
\hline Razones económicas & 62,4 & 42,2 \\
\hline Acceso a alimentos & 7,5 & 22,7 \\
\hline Reunificación familiar & 21,9 & 20,8 \\
\hline Acceso a medicinas/salud & 4,1 & 7,3 \\
\hline Falta de trabajo & 1,6 & 3,4 \\
\hline \multicolumn{3}{|l|}{ Tipo de grupo de viaje } \\
\hline Grupo familiar & 75,5 & 60,1 \\
\hline Sólo cuidador y niños & 12,5 & 21,7 \\
\hline Grupo no familiar & 7,5 & 5,8 \\
\hline Grupo mixto & 4,4 & 12,4 \\
\hline \multicolumn{3}{|l|}{ Duración del viaje } \\
\hline 3-9 días & 83,8 & 70,9 \\
\hline 10-30 días & 7,2 & 11,4 \\
\hline Un mes a más & 9,0 & 17,6 \\
\hline $\begin{array}{l}\text { Días (promedio/ } \\
\text { mediana/rango) }\end{array}$ & $20,2 / 5 / 3-594$ & $43,8 / 5 / 2-1290$ \\
\hline
\end{tabular}

La proporción de niños con anemia se encuentra dentro del rango de lo considerado como un problema de salud pública moderado ${ }^{(16)}$, sin embargo, por la naturaleza de esta afectación, no es plausible establecer que se deba a las condiciones del proceso migratorio.

Con base en estos resultados, la DIRESA Tumbes y UNICEF desarrollaron un conjunto de acciones destinadas
Tabla 3. Características de la lactancia y alimentación de niños inmigrantes venezolanos menores de dos años.

\begin{tabular}{lcc}
\hline Característica & $\begin{array}{c}\text { Ronda 1 } \\
(\mathbf{n = 1 6 0 )} \\
\mathbf{\%}\end{array}$ & $\begin{array}{c}\text { Ronda 2 } \\
\text { (n=160) } \\
\%\end{array}$ \\
\hline $\begin{array}{l}\text { Lactancia día previo } \\
\text { (según grupo etario) }\end{array}$ & \\
0-6 meses & & \\
6-11 meses & 91,3 & 95,5 \\
\hline 12-17 meses & 82,5 & 92,4 \\
\hline 18-23 meses & 71,1 & 74,5 \\
\hline Total & 28,6 & 36,1 \\
\hline $\begin{array}{l}\text { Variación de la lactancia en } \\
\text { el viaje }\end{array}$ & 68,8 & 71,7 \\
\hline Dejó de darle de lactar & 7,5 & \\
\hline Disminuyó la frecuencia & 6,7 & 0,0 \\
\hline La mantuvo igual & 27,5 & 19,1 \\
\hline Aumentó la frecuencia & 58,3 & 34,0 \\
\hline Frecuencia mínima de & & 47,0 \\
alimentación* \\
(según grupo etario)
\end{tabular}

* Cuando el niño que lacta consumió un mínimo de preparaciones espesas o sólidas para su edad el día previo al estudio: dos veces en las edades de 6 y 8 meses y tres veces entre 9 a 24 meses; en los no lactantes la frecuencia debió ser cuatro veces al día.

a proteger la salud y nutrición de los niños migrantes, tal como se detallan a continuación.

\section{EVALUACIÓN Y CONSEJERÍA NUTRICIONAL}

Con base en los resultados de la primera ronda, UNICEF en coordinación con la DIRESA Tumbes, establecieron un módulo de salud y nutrición dentro del CEBAF para atender a los niños migrantes menores de cinco años. El módulo se implementó en colaboración con la ONG PRISMA. En este módulo se ofrecieron evaluación y consejería nutricional, derivación a atención de salud de ser necesario y suplementación alimentaria. Los niños identificados durante la evaluación con DA u otros problemas de salud fueron referidos para el seguimiento y el tratamiento dentro del CEBAF al módulo de la Cruz Roja, donde hay personal médico especializado para esta atención. La consejería nutricional se enfocó en la promoción de la lactancia materna y prácticas apropiadas de alimentación complementaria. Entre septiembre 2018 y mayo 2019, UNICEF y PRISMA, evaluaron el estado nutricional de 9482 menores de cinco años (4787 niñas y 4695 niños), brindaron consejería en alimentación infantil a 6971 familias y derivaron a 1341 niños y niñas a atención en salud.

\section{SUPLEMENTOS ALIMENTICIOS}

Desde diciembre 2018 hasta mayo 2019 , se proporcionaron suplementos alimenticios a las mujeres lactantes con 
niños menores de dos años (1378) y niños y niñas de 6 a 59 meses (3111), respectivamente. Los niños y niñas recibieron cinco dosis diarias de un alimento suplementario listo para usar (RUTF por sus siglas en inglés, Plumpy $\mathrm{Doz}^{\mathrm{TM}}$ ) y las mujeres lactantes recibieron un suplemento alimenticio que consiste en barras energéticas adquiridas localmente para que puedan complementar su dieta. Estos suplementos tienen el objetivo de prevenir la desnutrición aguda, recuperándolos de su viaje reciente y apoyar su viaje en curso. En coordinación con la DIRESA Tumbes, también se proporcionaron multimicronutrientes y antiparasitarios (estos últimos a toda la familia).

\section{TRATAMIENTO Y PREVENCIÓN DE MORBILIDADES, Y OTRASACCIONES}

Para apoyar la recuperación de los niños que presenten enfermedad diarreica aguda, UNICEF proporcionó suplementos de zinc que se distribuyeron a través de la Cruz Roja y de seis establecimientos de salud cercanos al CEBAF. Asimismo, UNICEF y otras agencias dentro del CEBAF, en coordinación con la DIRESA Tumbes, brindaron, a través de la ONG COOPI (Cooperazione Internazionale), kits de cuidado e higiene básico dirigidos a niños y niñas menores de tres años. Hasta mayo 2019, se habían entregado 7837 kits. Además, se brindó apoyo psicoemocional y de gestión de crisis a madres, padres, cuidadores y niños que lo requerían.

\section{CONCLUSIONES Y RECOMENDACIONES}

Los resultados muestran que el estado nutricional de los niños migrantes podríaestaren riesgo por las condiciones del proceso migratorio, por lo que las acciones implementadas al momento de su ingreso al país estuvieron destinadas a proteger su salud y nutrición. Contar con información oportuna para la toma de decisiones en un contexto de migración dinámica, con un volumen y complejidad que supera los escenarios planificados, es un desafío que requiere una coordinación y despliegue rápidos.

Los flujos migratorios no son nuevos en la región, sin embargo, la magnitud del flujo migratorio venezolano es inédito para América Latina. El sistema de salud en el Perú, tanto en el ámbito nacional como subnacional, requiere incorporar estos escenarios en sus procesos de planeamiento y preparación a fin de poder afrontar estas circunstancias. Sin bien es cierto que la reacción oportuna frente al ingreso de la población migrante es un buen primer paso, el sistema de salud necesita generar las condiciones para que los servicios de salud puedan brindar la cobertura con la calidad adecuada a toda la población que reside en el territorio nacional, bajo el enfoque de que el derecho a la salud corresponde a todas las personas sin ningún tipo de exclusión.

Fuentes de financiamiento: Fondo de las Naciones Unidas para la Infancia (UNICEF).

Declaración de conflictos de interés: Ninguno de los autores tiene conflictos de interés. El manuscrito es de entera responsabilidad de los autores y no representa la posición de UNICEF.

\begin{abstract}
Contribuciones de autoría: MRVMA, CERD, MEUV participaron en la concepción del artículo. MRVMA, CPM participaron en la recolección de la información. MRVMA y CERD realizaron el procesamiento y análisis de los datos. CERD, MRVMA, MMJ realizaron la redacción del artículo. Todos los autores realizaron la revisión crítica del artículo y aprobaron la versión final.
\end{abstract}

\begin{abstract}
Agradecimientos: A la Dirección Regional de Salud de Tumbes, las ONG PRISMA y COOPI por el trabajo desplegado de manera conjunta con UNICEF para la implementación de las acciones de salud y nutrición; así como por las coordinaciones realizadas para llevar a cabo las evaluaciones nutricionales en el CEBAF. También agradecemos al Centro Nacional de Alimentación y Nutrición (CENAN) por su soporte técnico en el entrenamiento al personal de campo que llevó a cabo el levantamiento de la información, y por los instrumentos y equipamiento facilitados para realizar las mediciones antropométricas y el dosaje de hemoglobina.
\end{abstract}

\section{REFERENCIAS BIBLIOGRÁFICAS}

1 Carrasco I, Suárez J. Migración internacional e inclusión en América Latina: Análisis en los países de destino mediante encuestas de hogares [Internet]. Santiago: Naciones Unidas Comisión Económica para América Latina y el Caribe (CEPAL); 2019 [citado el 17 de junio de 2019]. Disponible en: https:// www.cepal.org/es/publicaciones/43947migracion-internacional-inclusionamerica-latina-analisis-paises-destino

2 Organización Internacional del Trabajo. Diagnóstico, estrategia y líneas de trabajo de la OIT en la Región. Diagnóstico, Estrategia y Líneas de Trabajo de la OIT en la Región [Internet]. Lima: Oficina
Regional para América Latina y el Caribe; 2016 [citado el 17 de junio de 2019]. Disponible en: https://www.lo.org/ wcmsp5/groups/public/---americas/--ro-lima/documents/publication/ wcms_502766.pdf

3 Burrone S, D'costa B, Holmqvist G. Child-Related Concerns and Migration Decisions: Evidence from the Gallup World Poll [Internet]. Florence: UNICEF Office of Research - Innocenti; 2018. [citado el 17 de junio de 2019]. Disponible en: https://www.unicef-irc. org/publications/1014-child-relatedconcerns-and-migration-decisionsevidence-from-gallup-world-poll.html
4 UNHCR-IOM. Coordination platform for refugees and migrants from Venezuela [Internet]. R4v.info; 2019 [citado el 5 de junio de 2019] . Disponible en: https:// r4v.info/es/situations/platform

5 UNICEF Latin America and the Caribbean Regional Office. Migration flows in Latin America and the CaribbeanSituation Report No. 4 [Internet]. Panamá: UNICEF; 2019 [citado el 17 de junio de 2019]. Disponible en: https://www. unicef.org/appeals/files/Latin_America_ Migration_Flows_May_2019.pdf

6 Organización Internacional para las Migraciones (OIM), Fondo de las Naciones 
Unidas para la Infancia (UNICEF). Monitoreo de Flujo de Población Venezolana en el Perú (DTM Ronda 4) [Internet]. Lima; 2018 [citado el 17 de junio de 2019]. Disponible en: https://r4v. info/es/documents/details/68475

7 Organización Internacional para las Migraciones (OIM), Fondo de las Naciones Unidas para la Infancia (UNICEF). Monitoreo de Flujo de Población Venezolana en el Perú (DTM Ronda 5) [Internet]. Lima; 2019 [citado el 17 de junio de 2019]. Disponible en: https://r4v. info/es/documents/details/68475

8 Bowers D, House A, Owens D. Getting Started in Health Research. Chichester, West Sussex, U.K.: Wiley-Blackwell/BMJ Books; 2011.

9 Van den Broeck J, BrestoffJ. Epidemiology: Principles and Practical Guidelines. Dordrecht: Springer Netherlands; 2013.

10 Word Health Organization. Physical status: the use and interpretation of anthropometry: report of a WHO Expert Committee-Series 854 [Internet]. Geneva: World Health Organization; 1995 [citado el 17 de junio de 2019]. Disponible en: https://apps.who.int/ iris/bitstream/handle/10665/37003/
WHO_TRS_854.pdf;jsession$\mathrm{id}=41 \overline{\mathrm{D}} 0 \mathrm{~A} 4 \mathrm{~F} 0604 \mathrm{~A} 9 \mathrm{~B} 9 \mathrm{ABB}$. 94B5A2AD338085? sequence $=1$

11 de Onis M. WHO child growth standards. Length/height-for-age, weight-for-age, weight-for-length, weight-for-height and body mass index-for-age Methods and development. Geneva: World Health Organization, Department of Nutrition for Health and Development; 2006.

12 World Health Organization. Nutritional anaemias: tools for effective prevention and control [Internet]. Geneva: World Health Organization; 2017 [citado el 17 de junio de 2019]. Disponible en: https://apps.who.int/ iris/bitstream/handle/10665/259425/ 9789241513067-eng.pdf ?sequence $=1$

13 World Health Organization. Indicators for assessing infant and young child feeding practices: conclusions of a consensus meeting held 6-8 November 2007 in Washington D.C., USA. Washington, D.C: World Health Organization; 2008.

14 World Health Organization. Anthro V3.2. Geneva: World Health Organization; 2018 [citado el 17 de junio de 2019]. Disponible en: http://www.who.int/ childgrowth/software/en/
15 United Nations Children's Fund, World Health Organization \& World Bank Group. Levels and trends in child malnutrition: key findings of the 2019 Edition of the Joint Child Malnutrition Estimates [Internet]. WHO/NMH/ NHD; 2019 [citado el 17 de junio de 2019]. Disponible en: https://www. who.int/nutgrowthdb/jme-2019-keyfindings.pdf

16 Levels and trends in child malnutrition: key findings of the 2019 Edition of the Joint Child Malnutrition Estimates [Internet]. Ginebra: World Health Organization; 2019 [citado el 17 de junio de 2019]. Disponible en: https:// www.who.int/nutgrowth db/jme2019-key-findings.pdf

Correspondencia: M. Rocio Vargas-Machuca Araujo

Dirección: Parque Melitón Porras 350,

Miraflores, Lima, Perú.

Tel: +51998410869

Correo electrónico:rvargasmachuca@unicef.org 